\title{
Designing Highly Stable Poly(sarcosine)-based Telodendrimer Micelles with High Drug Content Exemplified with Fulvestrant
}

\author{
Qing $\mathrm{Yu}^{1}$, Richard M. England ${ }^{1 *}$, Anders Gunnarsson $^{2}$, Robert Luxenhofer ${ }^{4,5}$, Kevin \\ Treacher ${ }^{3}$, Marianne B. Ashford ${ }^{1}$, \\ ${ }^{1}$ Advanced Drug Delivery, Pharmaceutical Sciences, R\&D, AstraZeneca, Macclesfield, SK10 2NA, UK \\ ${ }^{2}$ Discovery Sciences, R\&D, AstraZeneca, Gothenburg, 431 50, Sweden \\ ${ }^{3}$ New Modalities and Parenterals Development, Pharmaceutical Technology and Development, Operations, \\ AstraZeneca, Macclesfield, SK10 2NA, UK \\ ${ }^{4}$ Functional Polymer Materials, Institute for Functional Materials and Biofabrication, Department of Chemistry \\ and Pharmacy, Würzburg University, Röntgenring 11, 97070 Würzburg, Germany \\ ${ }^{5}$ Soft Matter Chemistry, Department of Chemistry and Helsinki Institute of Sustainability Science, Faculty of \\ Science, University of Helsinki, 00014 Helsinki, Finland
}




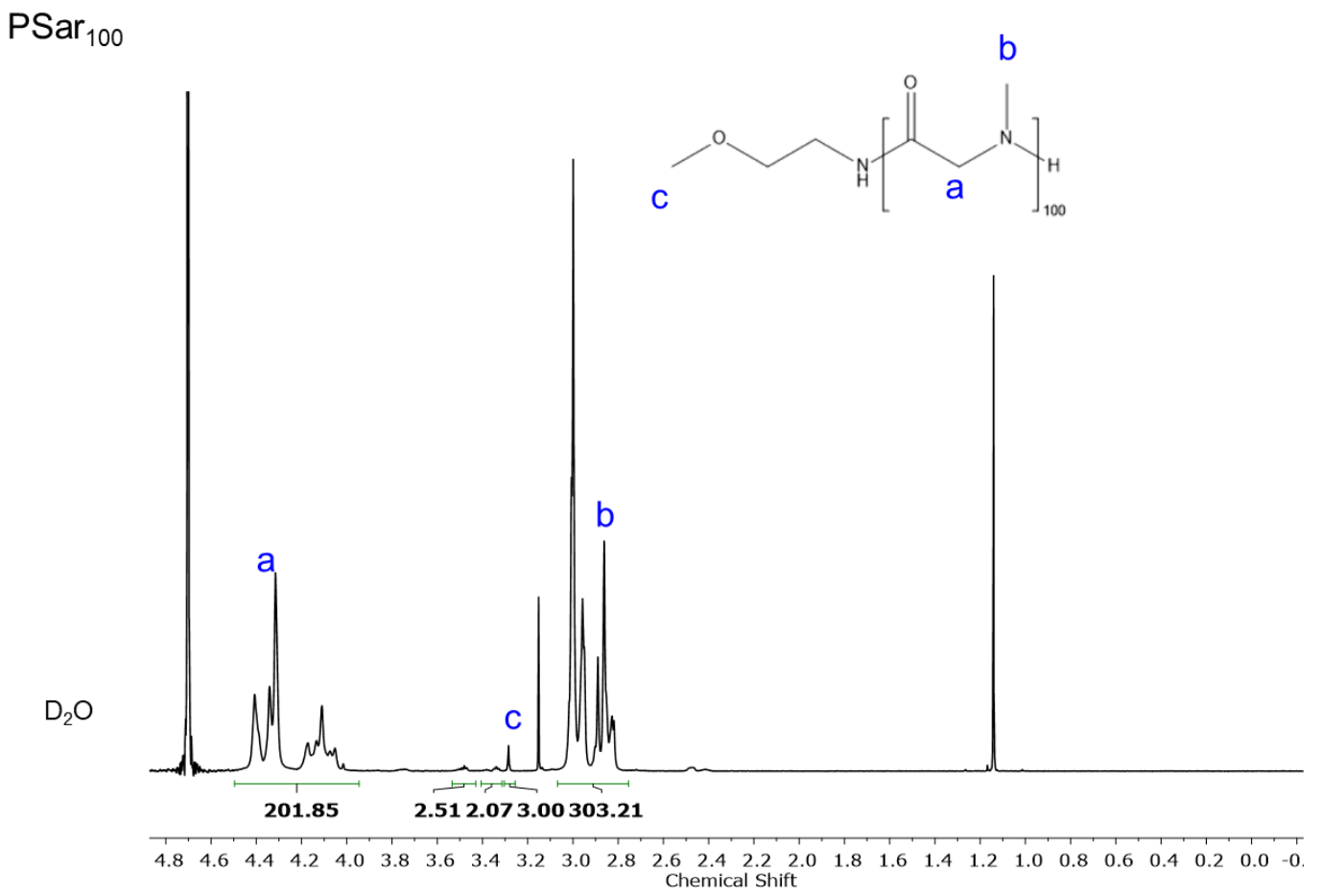

Figure S1. ${ }^{1} \mathrm{H}$ NMR spectrum of linear PSar ${ }_{100}$ in $\mathrm{D}_{2} \mathrm{O}$. 
(a)
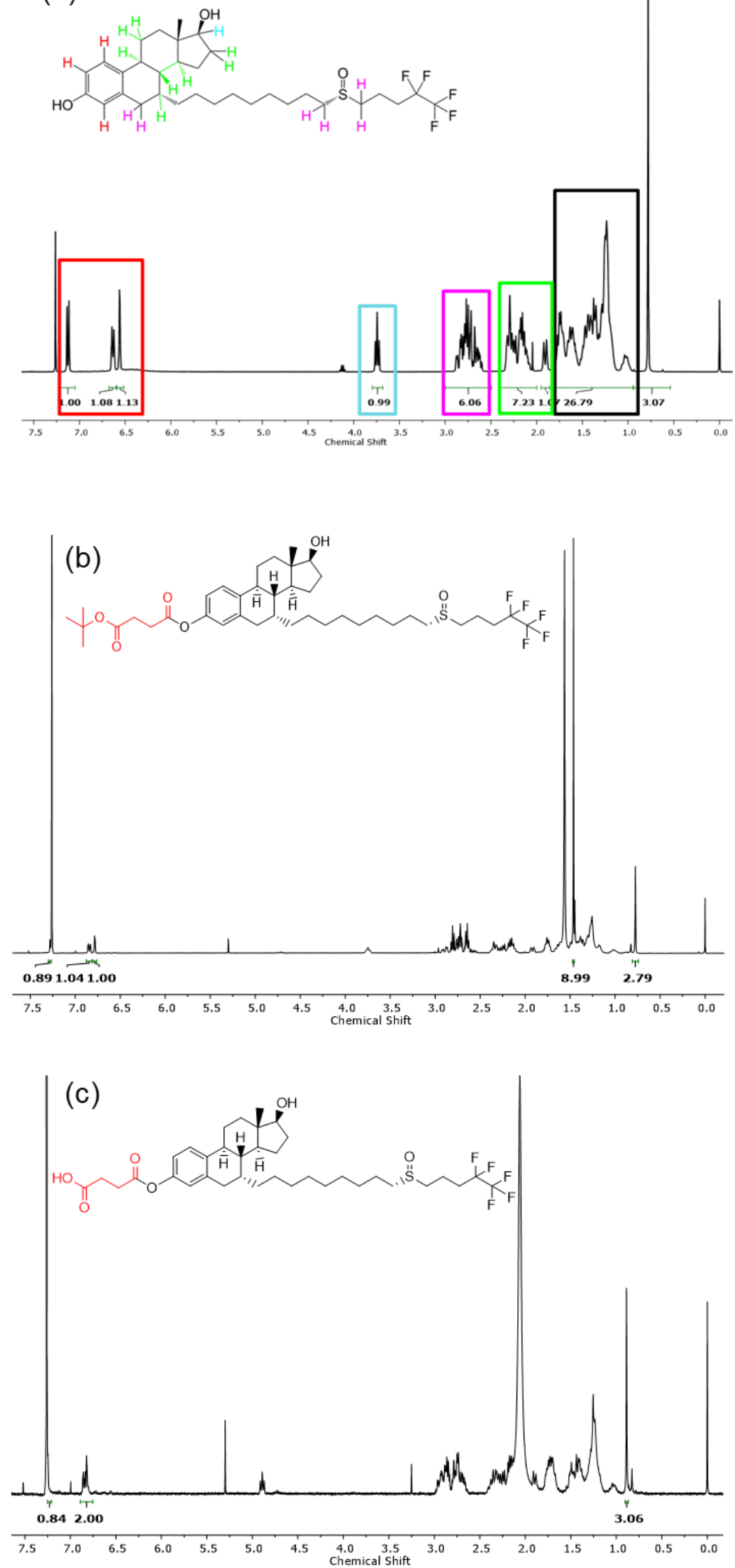

Figure S2. ${ }^{1} \mathrm{H}$ NMR spectra of (a) fulvestrant, (b) fulvestrant $t$-butyl succinate and (c) fulvestrant succinate in $\mathrm{CDCl}_{3}$. The disappearance of the sharp singlet peak at $1.5 \mathrm{ppm}$ indicated the complete removal of $t$-butyl protecting group. 

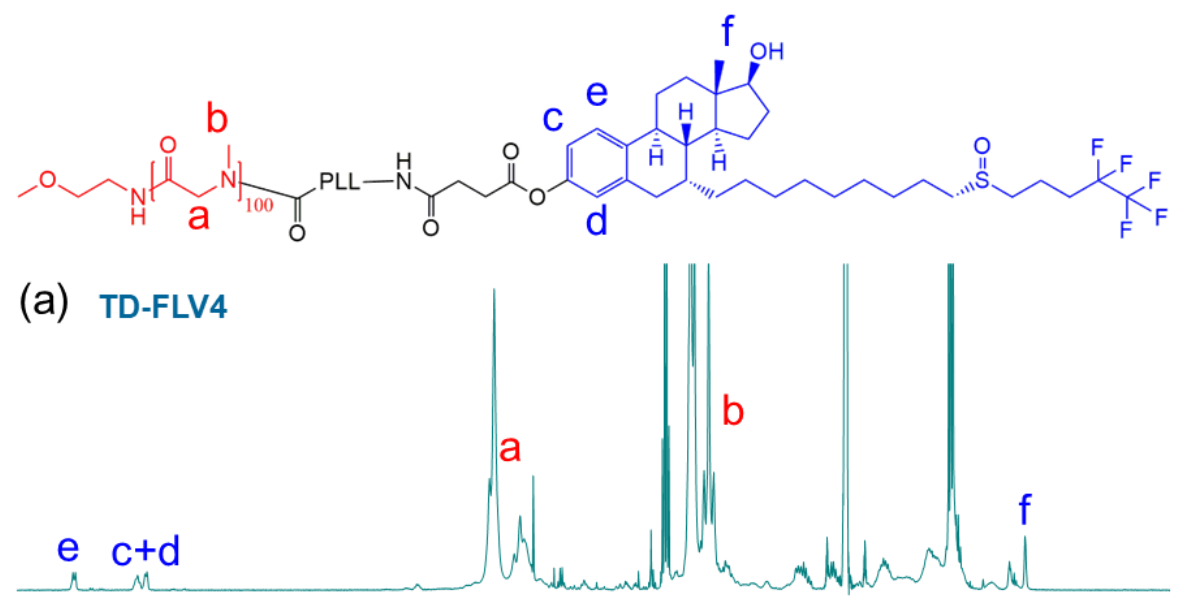

(b) TD-FLV16

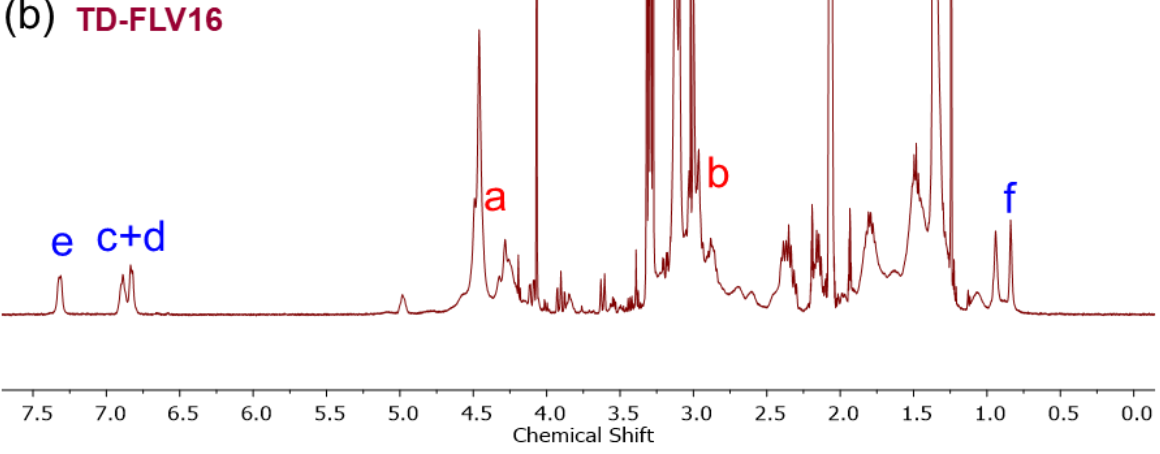

Figure S3. ${ }^{1} \mathrm{H}$ NMR spectra of (a) TD-FLV4 and (b) TD-FLV16 in $d$-acetic acid. The ratios between $e$ and $a$ are 4:200 and 16:200, respectively. 


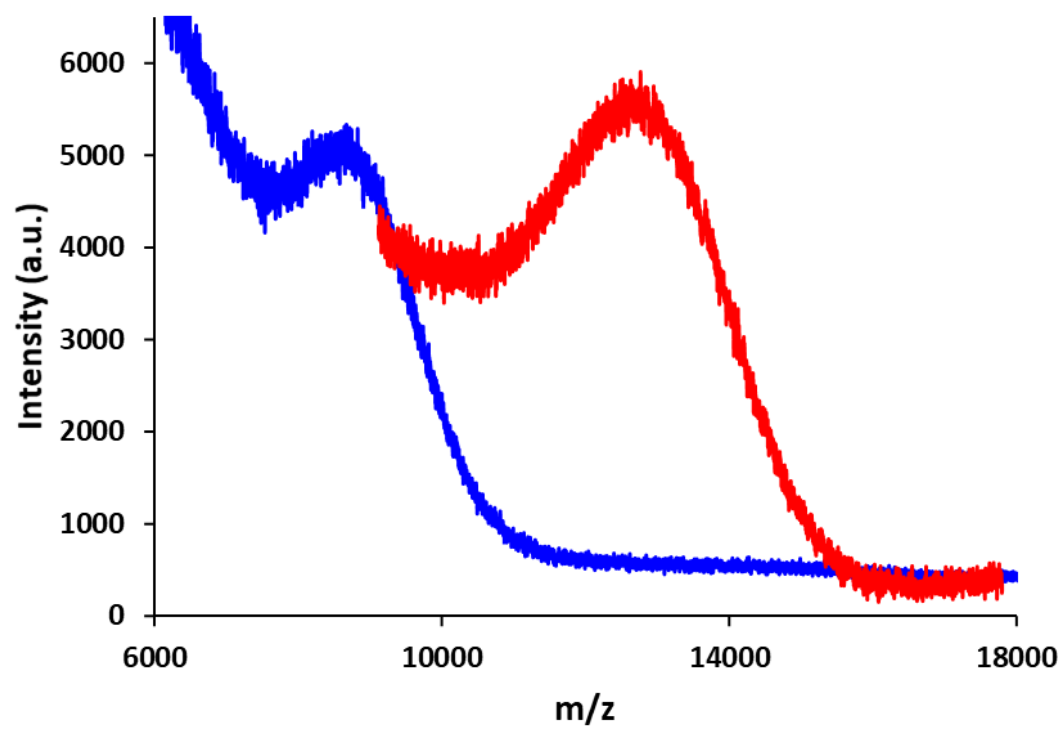

Figure S4. MALDI-TOF-MS spectra of TD-FLV4 (blue) with mass of $7 \mathrm{kDa}$ and TD-FLV8 (red) with mass of $13 \mathrm{kDa}$. 


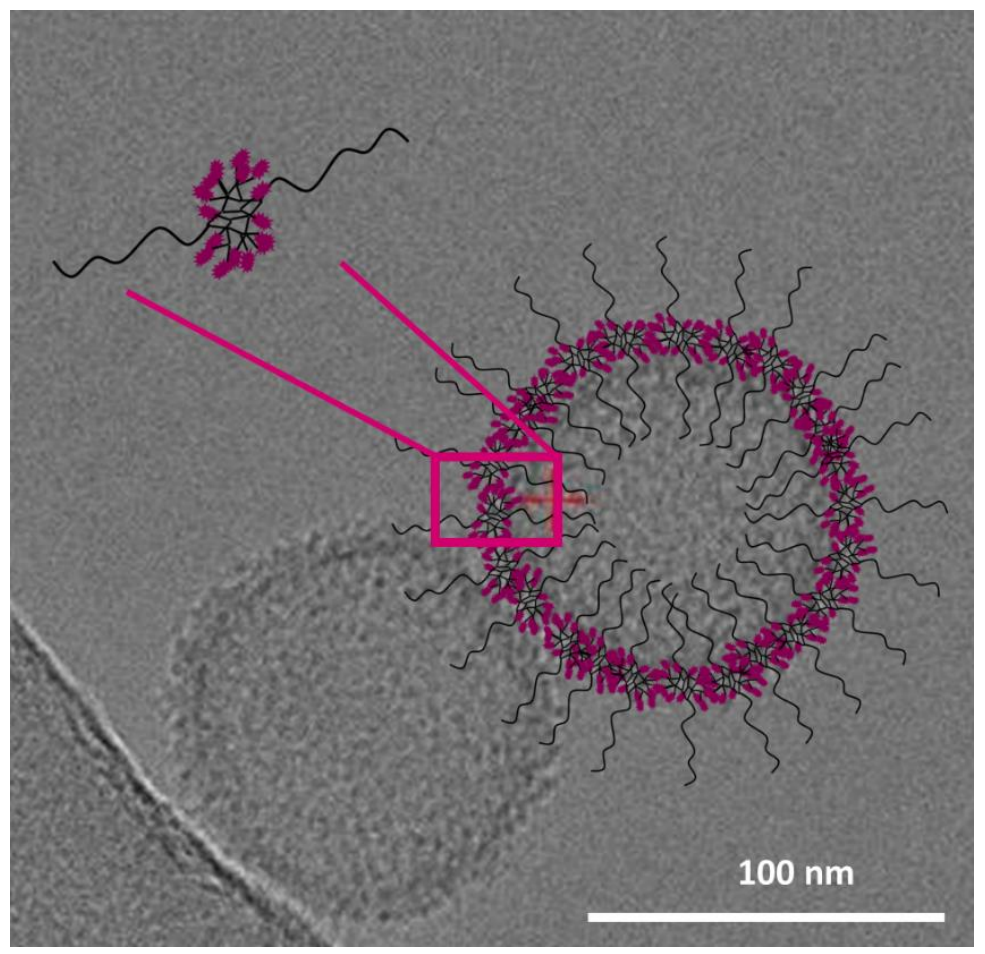

Figure S5. Cryo-EM image showing size, morphology and proposed unimer arrangement of TD-FLV16 micelle. Scale bar $=100 \mathrm{~nm}$. Images were collected using a Titan Krios G2 at $300 \mathrm{kV}$ equipped with a Falcon 2 direct electron detector (Thermo Fisher Scientific, Hillsboro, OR, USA) at a nominal magnification of $75 \mathrm{kx}$ ( $1 \AA /$ pixel) using EPU software with an electron fluence of $\sim 50 \mathrm{e} / \AA 2$. 


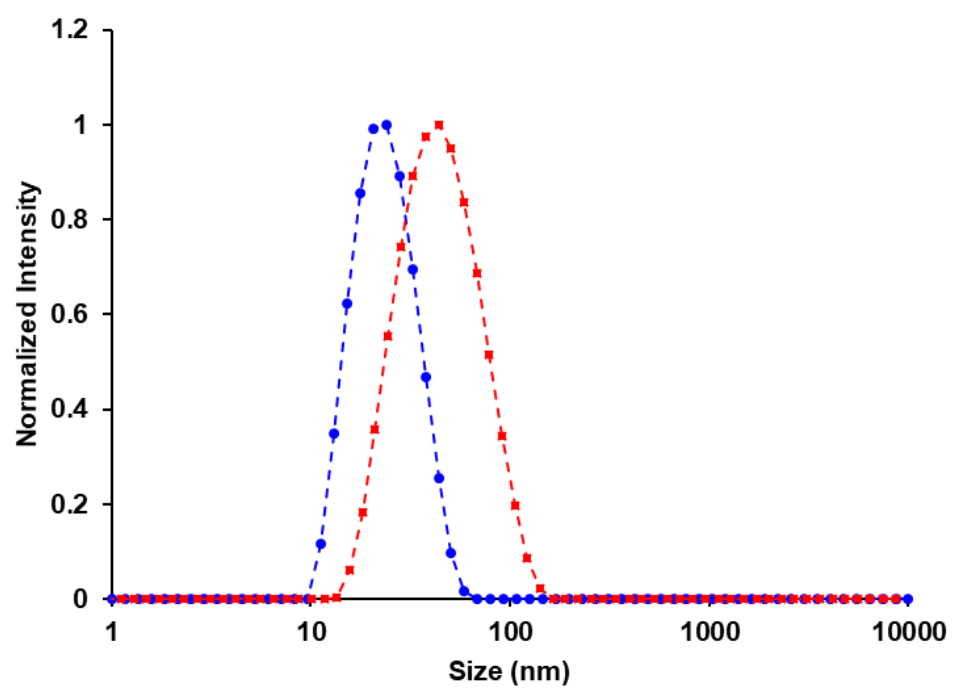

Figure S6. DLS of TD-FLV4 micelles prepared via (blue) co-solvent evaporation and (red) thin-film method in water. 


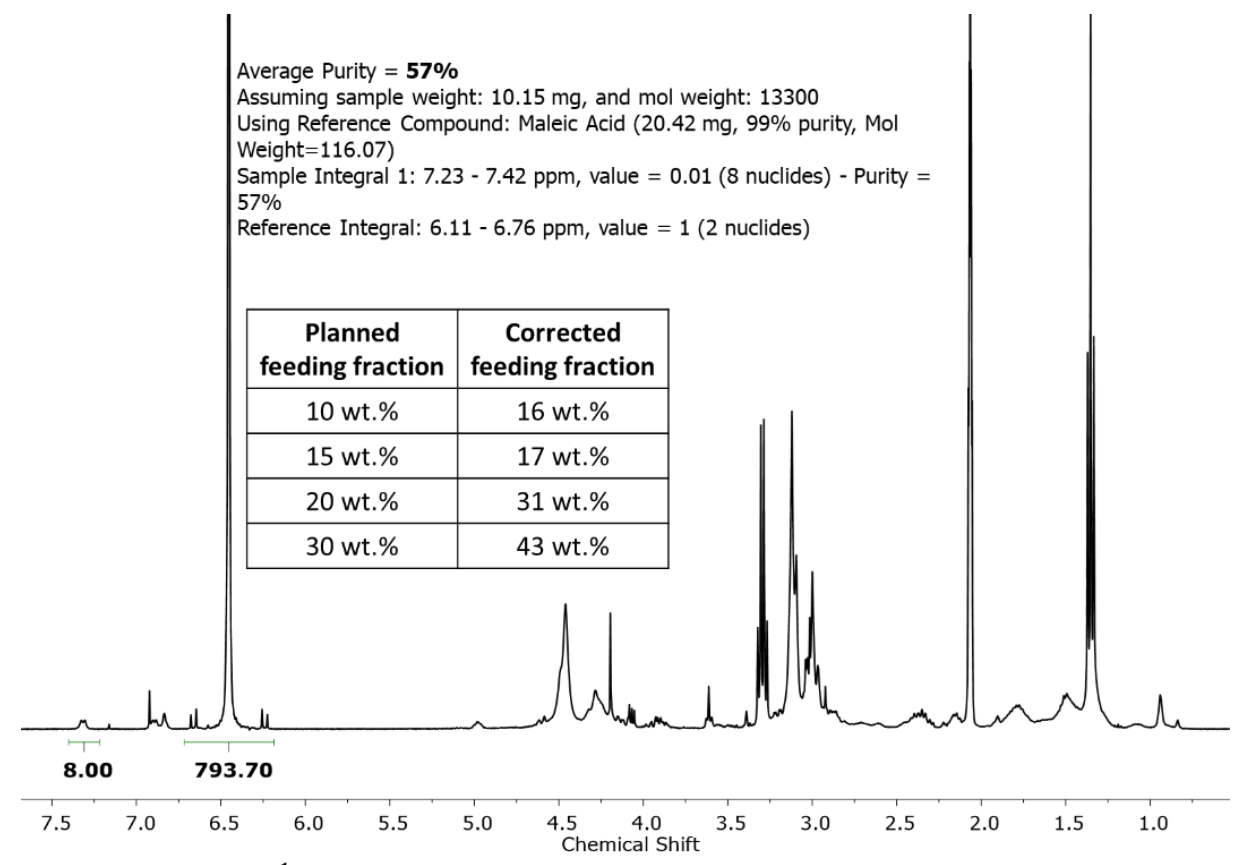

Figure S7. Quantification ${ }^{1} \mathrm{H}$ NMR spectrum of TD-FLV8 telodendrimer in $d$-acetic acid. The average purity was calculated as $57 \%$ using maleic acid as reference standard. The feeding fractions used to prepare fulvestrant-encapsulating TD-FLV8 micelles were corrected and shown in the table. 


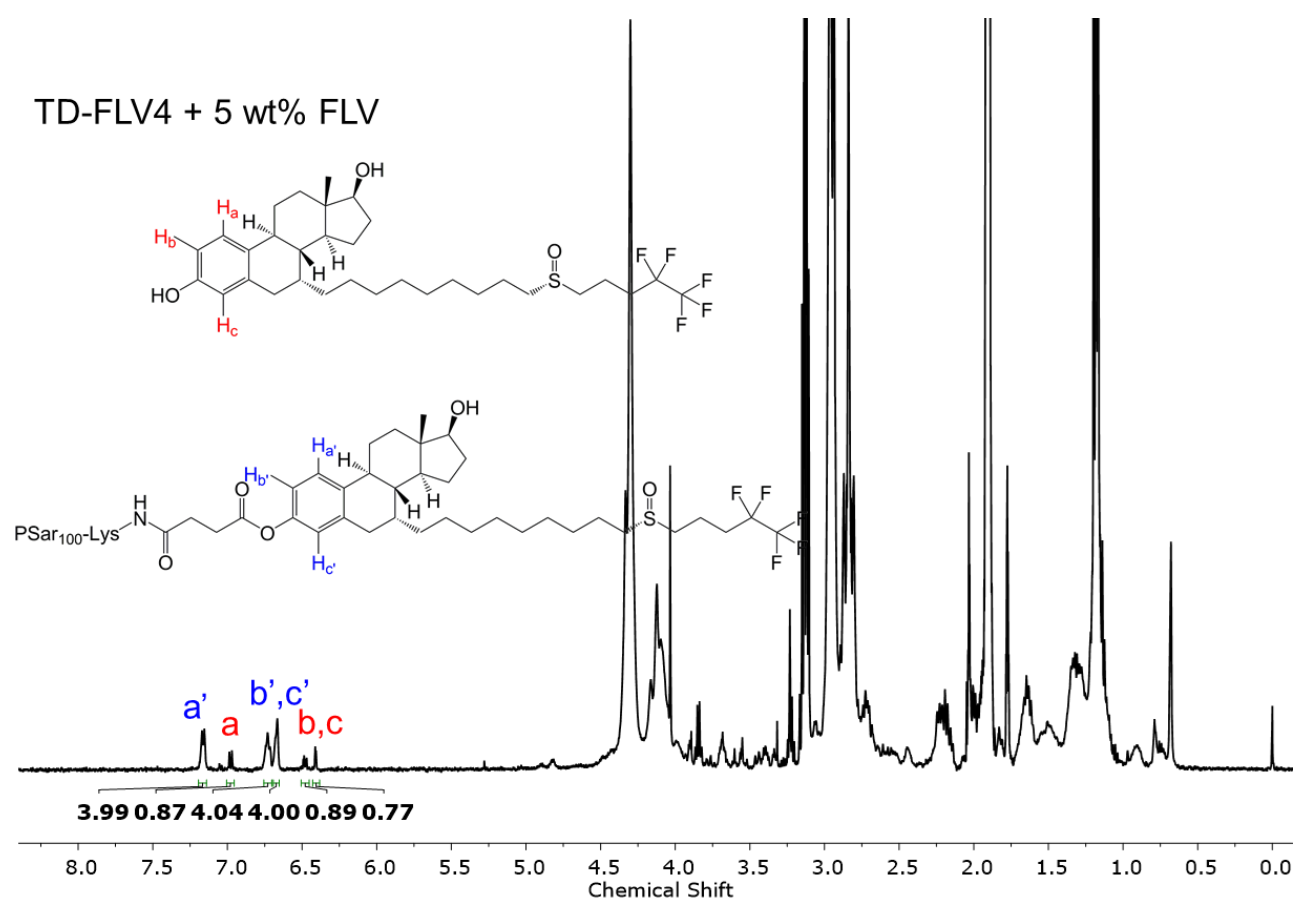

Figure S8. ${ }^{1} \mathrm{H}$ NMR spectrum of fulvestrant-loaded (5 wt.\%) TD-FLV4 micelle in $d$-acetic acid. The aromatic peaks ( $a, b$, and $c$ ) from fulvestrant broaden once conjugated to the telodendrimer ( $\mathrm{a}$ ', b', and c'). The ratio between $a$ and $a^{\prime}$ was used to determine the drug loading capacity. 


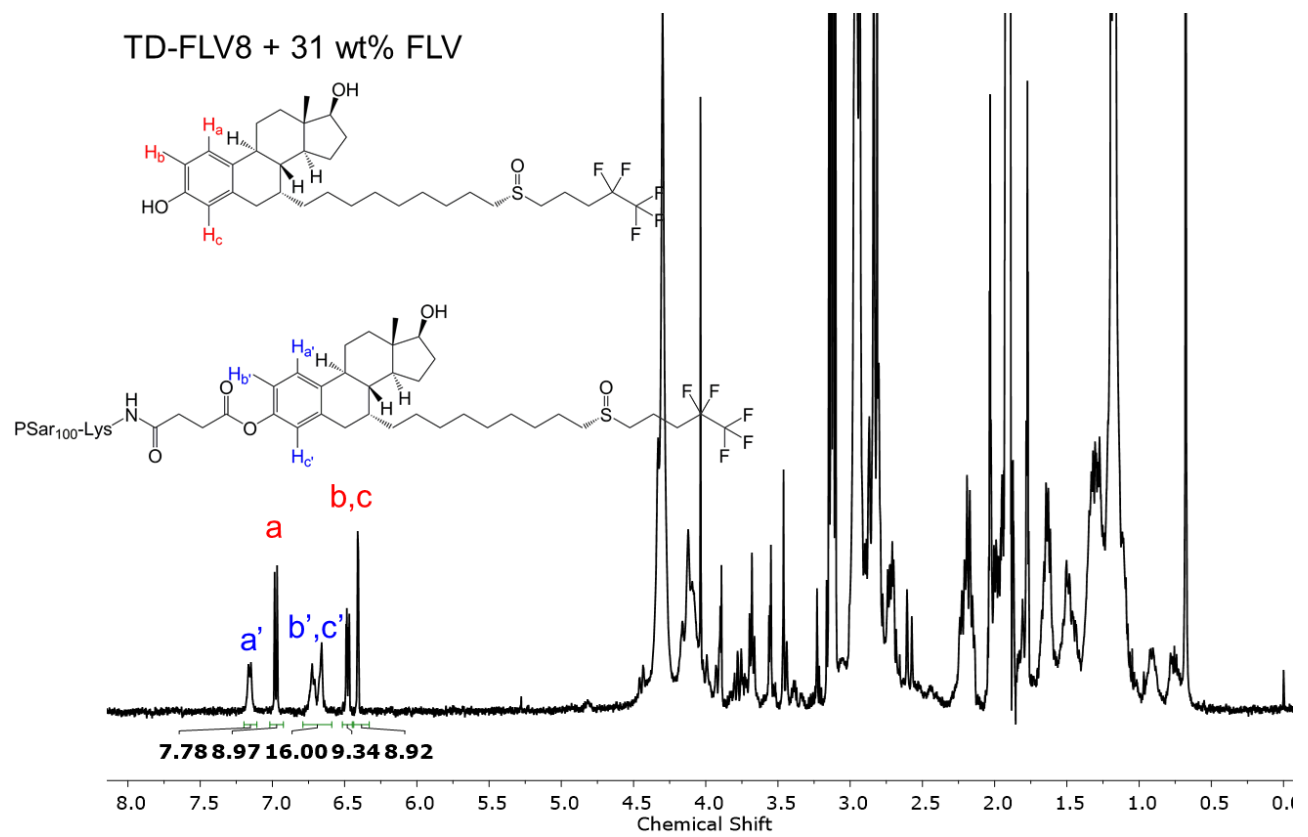

Figure S9. ${ }^{1} \mathrm{H}$ NMR spectrum of fulvestrant-loaded (31 wt.\%) TD-FLV8 micelle in $d$-acetic acid. The aromatic peaks ( $a, b$, and $c)$ from fulvestrant broaden once conjugated to the telodendrimer ( $\mathrm{a}^{\prime}, \mathrm{b}$ ', and c') and can be used to calculate the ratio between encapsulated vs. conjugated drug. 


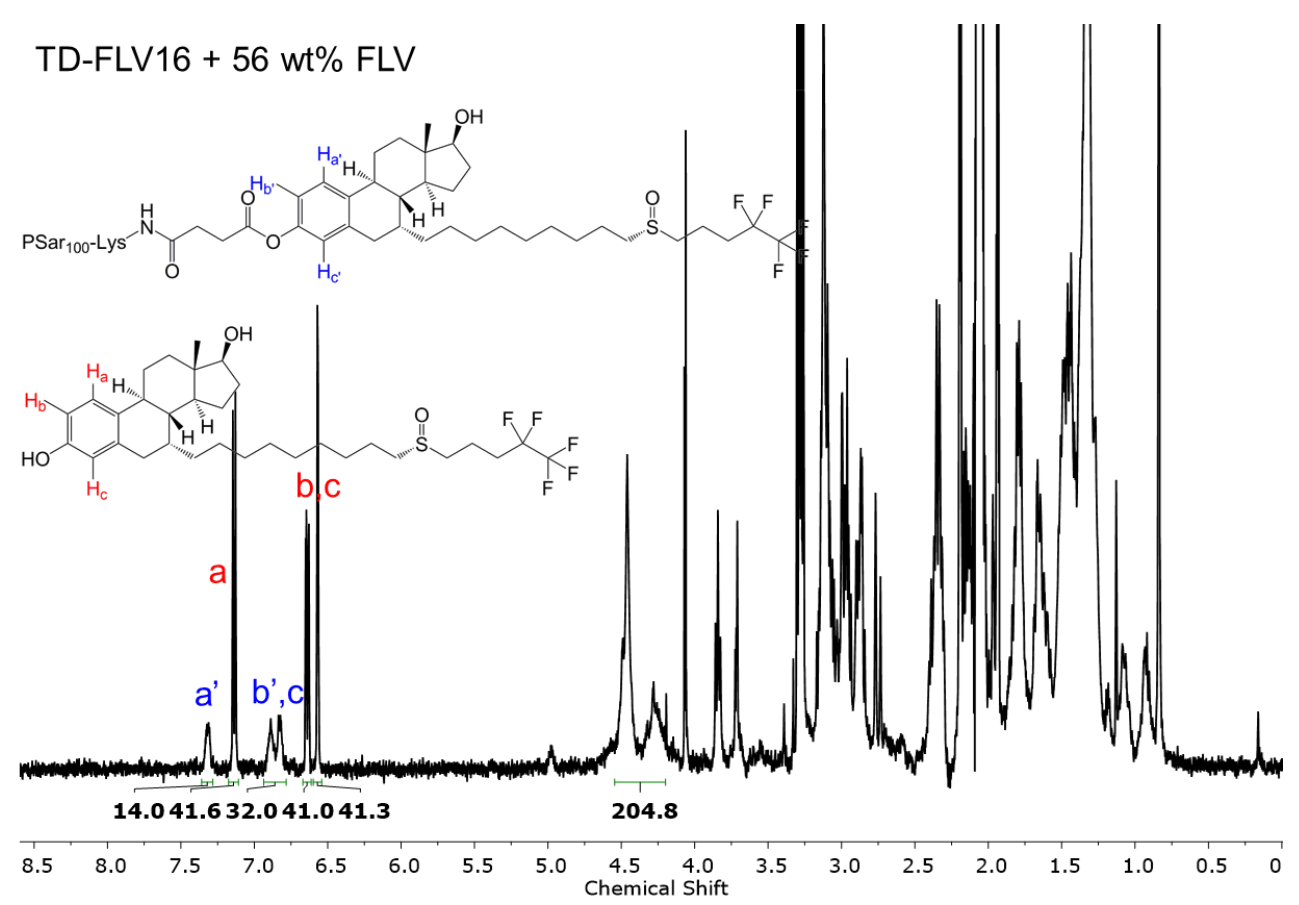

Figure S10. ${ }^{1} \mathrm{H}$ NMR spectrum of fulvestrant-loaded (56 wt. \%) TD-FLV16 micelle in $d$-acetic acid. The aromatic peaks ( $\mathrm{a}, \mathrm{b}$, and $\mathrm{c}$ ) from fulvestrant broaden once conjugated to the telodendrimer ( $\mathrm{a}^{\prime}, \mathrm{b}$ ', and c') and can be used to calculate the ratio between encapsulated vs. conjugated drug. 

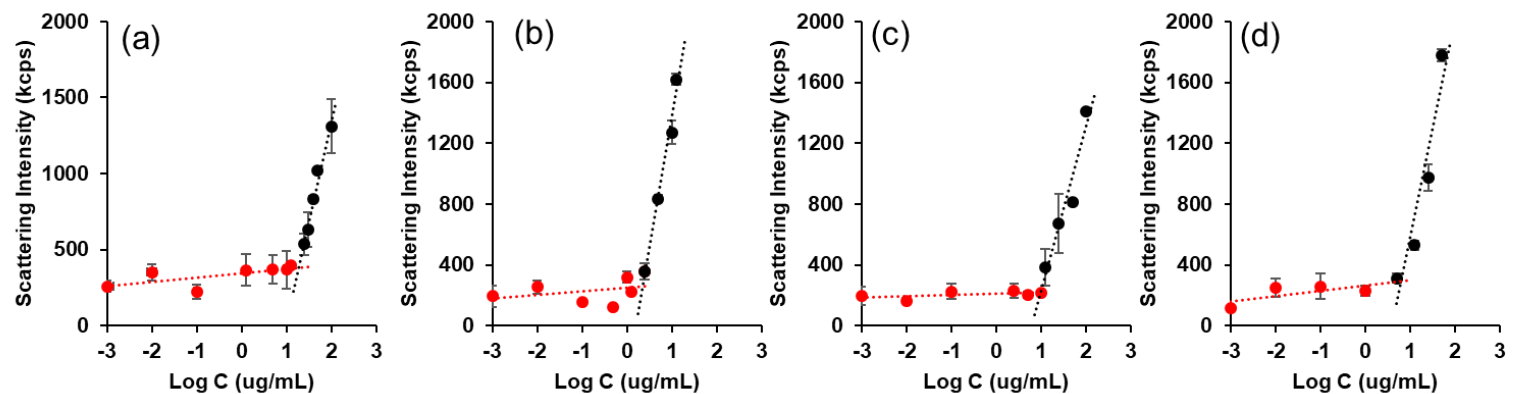

(e)
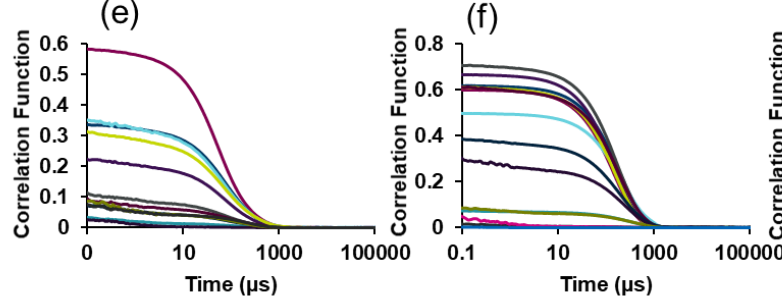

(g)

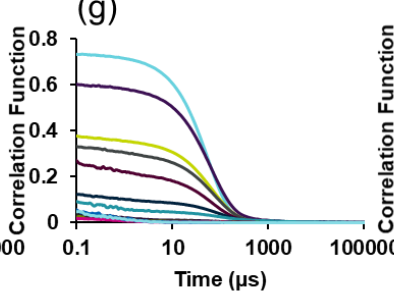

(h)

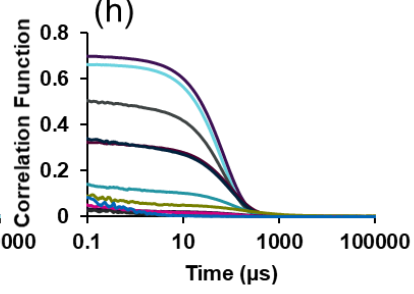

Figure S11. Determination of CMC using (a-d) scattering intensity and (e-h) autocorrelation

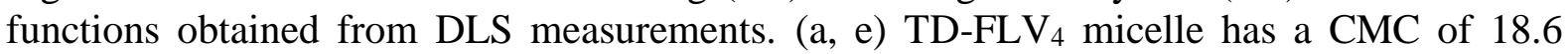
$\mu \mathrm{g} / \mathrm{mL}$, (b, f) TD-FLV 16 micelle has a CMC of $2.14 \mu \mathrm{g} / \mathrm{mL}$, (c, g) TD-FLV 8 micelle loaded with $10 \mathrm{wt} \%$ fulvestrant ( $\operatorname{size}=35 \pm 0.4 \mathrm{~nm}, \mathrm{PDI}=0.14$ ) has a CMC of $9.55 \mu \mathrm{g} / \mathrm{mL}$, and $(\mathrm{c}$, g) $\mathrm{TD}-\mathrm{FLV}_{8}$ micelle loaded with $20 \mathrm{wt} \%$ fulvestrant (size $=40 \pm 0.5 \mathrm{~nm}, \mathrm{PDI}=0.13$ ) has a $\mathrm{CMC}$ of $5.75 \mu \mathrm{g} / \mathrm{mL}$. 


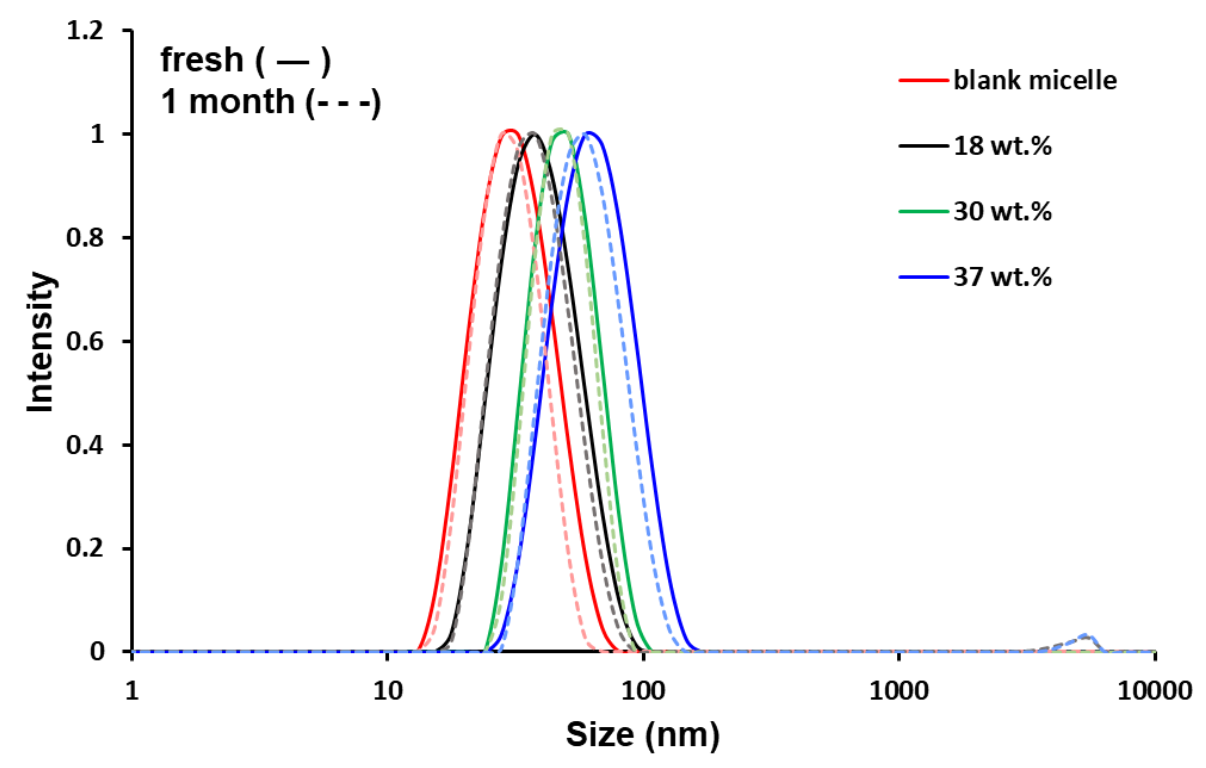

Figure S12. DLS data showing size changes of different telodendrimer micelles over 1 month time. Micelles were kept in water at $4{ }^{\circ} \mathrm{C}$ in the dark. 


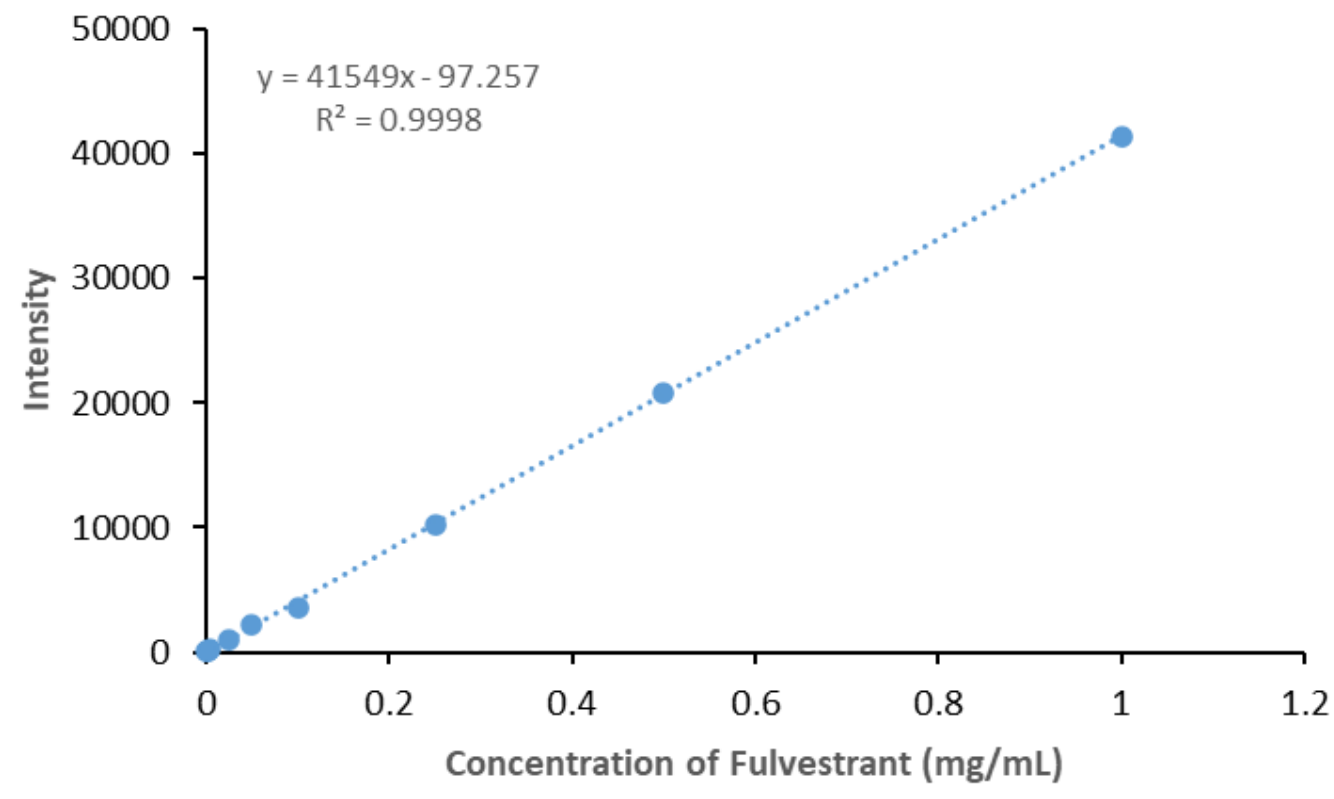

Figure S13. UPLC standard calibration curve of Fulvestrant in water/acetonitrile (1:1 by volume). 
Table S1. Experimental data of the in vitro drug release study showing the percentage release at each time point.

\begin{tabular}{ccccc}
\hline Time (h) & pH 7.4 & pH 7.4 + BSA & pH 5.5 & pH 8.5 \\
\hline 1 & $1.74 \pm 1.27$ & $2.34 \pm 1.73$ & $0.01 \pm 0.74$ & $9.11 \pm 2.14$ \\
4 & $4.17 \pm 0.46$ & $2.62 \pm 1.26$ & $0.01 \pm 0.94$ & $22.53 \pm 5.98$ \\
8 & $8.93 \pm 1.09$ & $5.44 \pm 3.22$ & $0.97 \pm 1.37$ & $36.21 \pm 10.55$ \\
24 & $21.20 \pm 0.89$ & $18.86 \pm 4.22$ & $1.33 \pm 0.12$ & $52.85 \pm 5.04$ \\
32 & $27.12 \pm 2.51$ & $19.19 \pm 0.42$ & $3.04 \pm 0.93$ & $61.69 \pm 6.78$ \\
48 & $33.33 \pm 4.14$ & $25.06 \pm 0.96$ & $2.81 \pm 0.47$ & $62.85 \pm 7.82$ \\
56 & $39.19 \pm 2.49$ & $32.26 \pm 1.93$ & $4.37 \pm 0.55$ & $69.45 \pm 10.08$ \\
72 & $40.08 \pm 3.67$ & $37.05 \pm 2.43$ & $3.56 \pm 1.27$ & $69.61 \pm 5.43$ \\
96 & $50.96 \pm 1.39$ & $43.25 \pm 2.07$ & $4.84 \pm 0.59$ & $78.09 \pm 5.85$ \\
168 & $60.84 \pm 1.02$ & $54.50 \pm 3.35$ & $8.70 \pm 2.14$ & $86.68 \pm 4.53$ \\
\hline
\end{tabular}

